\title{
Psoriatic skin lesions induced by tumor-necrosis-factor-alpha-therapy ${ }^{*}$
}

\author{
Antonia Cosima Faul ${ }^{1}$, Christian Folwaczny ${ }^{2}$, Birgit Angela Aigner ${ }^{3,4}$, Rüdiger Hein ${ }^{1}$, \\ Johannes Ring ${ }^{1}$, Sabine Gisela Plötz ${ }^{4 \#}$ \\ ${ }^{1}$ Department of Dermatology and Allergology, Technical University Munich, Munich, Germany \\ ${ }^{2}$ Gastroenterology Office, Medical Center Munich-Harlaching, Munich, Germany \\ ${ }^{3}$ Department of Dermatology, Medical University of Graz, Graz, Austria \\ ${ }^{4}$ Dermatology Munich-Harlaching, Munich, Germany; ${ }^{\#}$ Corresponding Author: hautarzt@aerztehaus-harlaching.de
}

Received 25 January 2013; revised 28 February 2013; accepted 14 March 2013

\begin{abstract}
A 46-year-old, caucasian woman suffering from Crohn's disease and a 35-year-old woman suffering from ankylosing spondylitis were treated with the TNFa blocker infliximab and developed psoriasiform skin lesions. The pathophysiology of this paradoxical clinical response as a newly recognized adverse effect of TNFa antagonists is unknown. According to the literature, this type of newly triggered psoriasis may occur any time after initiation of TNFa antagonist therapy and responds to classic anti-psoriatic treatment. The phenomenon warrants attention, especially when considering anti-TNF $\alpha$ treatment in various skin diseases besides psoriasis.
\end{abstract}

Keywords: Infliximab; Psoriasis; TNF $\alpha$ Inhibitors; Crohn's Disease; Ankylosing Spondylitis; Side Effects

\section{INTRODUCTION}

Tumor necrosis factor $\alpha$ (TNF $\alpha$ ) inhibitors, such as the monoclonal antibodies infliximab, adalimumab and the receptor antagonist etanercept, are used successfully for the therapy of several autoimmune disorders as well as diseases associated with a Th1 profile [secretion of interleukin-1, TNF $\alpha$ and interferon- $\gamma$ ]. Clinical efficacy of anti-TNF treatment has been established in rheumatoid arthritis, Crohn's Disease [1], ankylosing spondylitis [2] and in psoriasis [3].

Infliximab is a monoclonal human-mouse chimeric antibody that binds and inactivates TNF $\alpha$, an important proinflammatory cytokine. The chief adverse effect of $\mathrm{TNF} \alpha$ blockers is the elevated risk of infections.

In some single cases, more severe cutaneous reactions

"The authors declare no conflict of interest. like erythema multiforme, discoid and subacute lupus erythematosus, atopic eczema and seborrhoic dermatitis, necrotizing vasculitis and bullous skin lesions were observed [4-7]. One side effect is the new onset or acute exacerbation of psoriatic skin lesions [4,5,8-15]. In this article we present two cases of newly triggered psoriatic skin lesions in a patient with Crohn's disease and a further patient with ankylosing spondylitis after treatment with the monoclonal anti-TNF- $\alpha$ antibody infliximab.

\section{OBSERVATION}

\subsection{Case 1}

A 46-year-old, caucasian woman with a 20-year history of Crohn's disease had been treated with i.v. infliximab application $(3 \mathrm{mg} / \mathrm{kg})$ every 8 weeks over three years with good clinical response. There was no history of previous skin rash or allergic reactions but the patient reported erythema nodosum in context with Crohn's disease two years before initiation of infliximab therapy. Three years after initiation of treatment the patient developed an erythematosquamous eruption on the inguinal areas, the trunk, the elbows and the scalp (Figure 1). Skin biopsy showed akanthosis, elongated rete ridges and hyper- and parakeratosis (Figure 2). Therapy with topical steroids led to clearance of lesions, but after therapy cessation they reappeared on different localizations. Because of the good results regarding the Crohn's disease the patient tolerated the cutaneous side effects and decided to continue the therapy with infliximab.

\subsection{Case 2}

A 35-year-old, female patient, suffering from ankylosing spondylitis (AS) has been treated with i.v. infliximab $5 \mathrm{mg} / \mathrm{kg}$ every 6 weeks over 2 years with excellent clinical response. There was no history of previous side effects. 27 month after the initial treatment with infliximab the patient developed an erythematosquamous 


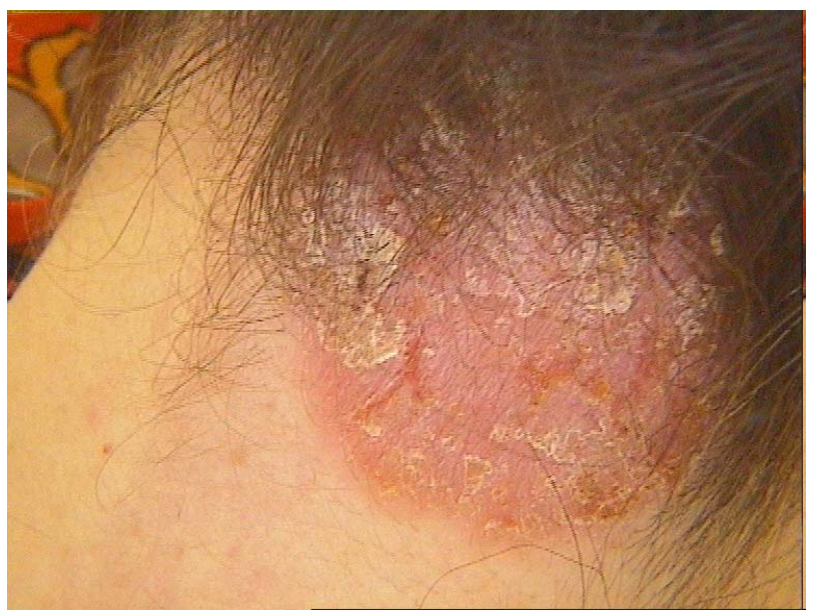

Figure 1. Psoriatic skin lesions on the neck induced by tumornecrosis-factor-alpha-therapy.

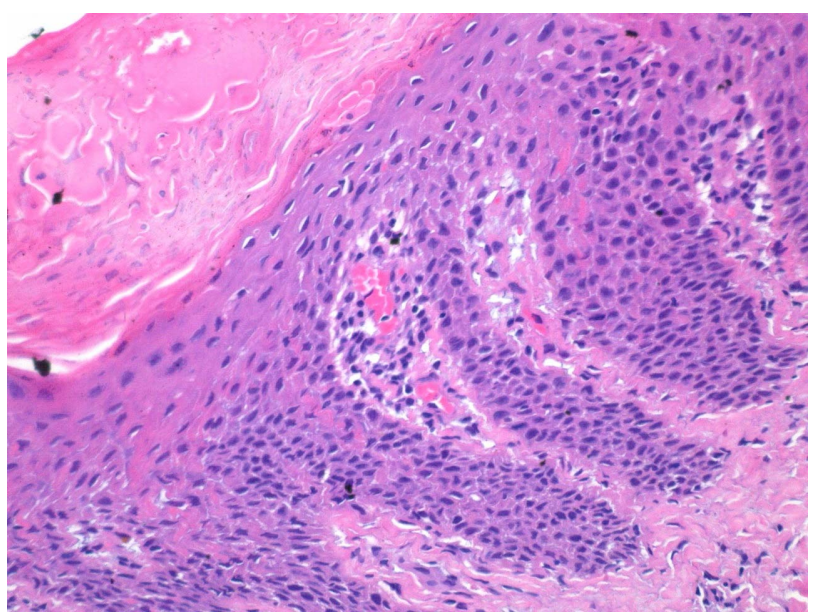

Figure 2. Psoriatic skin lesions induced by tumor-necrosisfactor-alpha-therapy: skin biopsy revealed akanthosis, elongated rete ridges and hyper and parakeratosis.

eruption on the capilitium and periorbital region as well as erythematous lesions on the vulva. Topical treatment with steroids resulted remission of the skin lesions. Because of the good clinical results regarding AS therapy with the TNF $\alpha$-inhibitor was continued.

\section{DISCUSSION}

We report two cases of newly triggered psoriasiform skin lesions in patients who had been treated with infliximab for Crohn's disease and ankylosing spondylitis.

Since the use of TNF antagonists has increased, it has been more and more apparent that a wide variety of adverse cutaneous reactions can be associated with this therapy $[6,7,14]$. However, a rather common side effect is the development or worsening of psoriasis. It is now generally accepted that this adverse effect can occur at any time from days to years after initiation or after treatment, independently of dosage and with all three available
$\mathrm{TNF} \alpha$ antagonists suggesting a class effect, rather than a drug-specific effect [15]. In most of the reported cases there seem to be more women affected, but this predilection is not statistically proven.

It seems paradox that anti-TNF agents-which are effective in the therapy of psoriasis - are able to aggravate or even induce the disease. This fact highlights the important and complex role of $\mathrm{TNF} \alpha$ for the pathogenesis of psoriasis, which is not yet completely understood.

An explanation could be the fact that prevalence of psoriasis may be higher among patients with chronic inflammatory conditions. In the study of Sfikakis et al., a cohort of 100 patients with chronic arthritic conditions was treated with infliximab, adalimumab and etanercept. During the follow-up, 5 patients developed psoriatic eruptions. The authors conclude that, although the prevalence of psoriasis may be higher among patients with chronic inflammatory arthritic conditions, its occurrence in 5\% of their patients who received anti-TNF treatment clearly exceeds the prevalence that might be expected by chance, especially given the short period of observation [9].

Review of the literature revealed several closely associated immunologic pathways that may induce this paradoxical reaction in a susceptible subset of patients. Plasmacytoid dendritic cells (PDCs) and their production of interferon- $\alpha$ (IFN $\alpha$ ) appear to be the key factors in psoriasis induction in these patients [11]. TNF $\alpha$ normally regulates IFN $\alpha$ production at two levels [16]. It inhibits PDC maturation from hematopoietic progenitors as well as IFN $\alpha$ release by activated PDCs. Thus, the inhibition of TNF $\alpha$ may allow unlimited and unregulated production of IFN $\alpha$ by PDCs [17]. Compared to healthy controls, PDCs are found in increased numbers in early psoriatic lesions and even in the normal-appearing skin of psoriatic patients [18]. A potential involvement of IFN $\alpha$ in the pathogenesis of psoriasis has been previously suggested by several observations $[19,20]$. Cutaneous application of imiquimod cream, a potent inducer of IFN $\alpha$, has been described to induce exacerbation of psoriasis [21]. Finally, increased IFN $\alpha$ expression has been demonstrated in patients who developed psoriatic lesions under TNF antagonist therapy [11]. This suggests an important role of IFN $\alpha$ in the pathogenesis of skin dysregulation in the setting of TNF inhibition.

Intriguingly, anti-TNF treatment has also been shown to enhance the expression of chemokine receptors, such as CXCR3 in circulating $\mathrm{T}$ cells, promoting infiltration of autoreactive cells to the skin. Aeberli et al. found a marked increase in CXCR3+ Th1-type T lymphocytes in peripheral blood following anti-TNF treatment and in patients with rheumatoid arthritis, IFN $\alpha$ has been shown to induce the expression of the chemokine receptor CXCR3 on $\mathrm{T}$ cells, facilitating their homing to the skin [17]. TNF $\alpha$ inhibition has also been shown to decrease traf- 
ficking of Th1 lymphocytes to the sites of joint destruction in autoimmune inflammatory disorders, resulting in an increase in the pooling and sequestration of these cells in the peripheral circulation. This T-cell population has the potential for activation and mobilization to cutaneous sites [15,17]. Thus, the inhibition of $\mathrm{TNF} \alpha$, resulting in an increased IFN $\alpha$ expression and homing of Th1 cells to the skin, might be a probable mechanism for the development of psoriasis in patients during TNF antagonist therapy.

Psoriasiform cutaneous side effects during anti-TNF $\alpha$ therapy are not exceptional and seem to be an anti-TNF $\alpha$ class effect in possibly genetically-predisposed individuals without evidence of a systematic related personal or family history of psoriasis. The clinical presentation is heterogeneous and most of the eruptions are self-limited or respond well to topical treatment. Unfortunately the wide variety of demographic characteristics, therapy concepts and different kinds of TNF $\alpha$ inhibitors makes it hard to formulate useful suggestions to prevent these adverse reactions. But as the use of $\mathrm{TNF} \alpha$ antagonists continues to increase, we must remain vigilant in the monitoring and reporting of adverse and unexpected reactions, with hope of increasing our knowledge about their pathophysiology.

In summary, we have described two cases of newly triggered psoriasis that occurred after repeated infusions of infliximab. The pathophysiology of this phenomenon is unclear, but the finding warrants attention, especially in a time when anti-TNF-treatment is increasingly used for a variety of skin diseases including psoriasis.

\section{REFERENCES}

[1] Keating, G.M. and Perry, C.M. (2002) Infliximab: An updated review of its use in Crohn's disease and rheumatoid arthritis. BioDrugs, 16, 111-148. doi:10.2165/00063030-200216020-00005

[2] Braun, J. and Sieper, J. (2004) Biological therapies in the spondyloarthritides-the current state. Rheumatology (Oxford), 43, 1072-1084. doi:10.1093/rheumatology/keh205

[3] Mease, P. (2004) TNFalpha therapy in psoriatic arthritis and psoriasis. Annals of the Rheumatic Diseases, 63, 755758. doi:10.1136/ard.2004.020719

[4] Stratigos, A.J., Antoniou, C., Stamathioudaki, S., et al. (2004) Discoid lupus erythematosus-like eruption induced by infliximab. Clinical and Experimental Dermatology, 29, 150-153. doi:10.1111/j.1365-2230.2004.01471.x

[5] Lebas, D., Staumont-Salle, D., Solau-Gervais, E., et al. (2007) Cutaneous manifestations during treatment with TNF-alpha blockers: 11 cases. Annales de Dermatologie et de Venereologie, 134, 337-342. doi:10.1016/S0151-9638(07)89187-3

[6] Flendrie, M., Vissers, W.H., Creemers, M.C., et al. (2005) Dermatological conditions during TNF-alpha-blocking therapy in patients with rheumatoid arthritis: A prospective study. Arthritis Research \& Therapy, 7, R666-R676. doi:10.1186/ar1724

[7] Devos, S.A., Van Den Bossche, N., De Vos, M. and Naeyaert, J.M. (2003) Adverse skin reactions to anti-TNFalpha monoclonal antibody therapy. Dermatology, 206, 388-390. doi:10.1159/000069965

[8] Starmans-Kool, M.J., Peeters, H.R. and Houben, H.H. (2005) Pustular skin lesions in patients treated with infliximab: Report of two cases. Rheumatology International, 25, 550-552. doi:10.1007/s00296-004-0567-5

[9] Sfikakis, P.P., Iliopoulos, A., Elezoglou, A., et al. (2005) Psoriasis induced by anti-tumor necrosis factor therapy: A paradoxical adverse reaction. Arthritis \& Rheumatism, 52, 2513-2518. doi:10.1002/art.21233

[10] Takahashi, H., Hashimoto, Y., Ishida-Yamamoto, A., et al. (2007) Psoriasiform and pustular eruption induced by infliximab. The Journal of Dermatology, 34, 468-472. doi:10.1111/j.1346-8138.2007.00312.x

[11] de Gannes, G.C., Ghoreishi, M., Pope, J., et al. (2007) Psoriasis and pustular dermatitis triggered by TNF-\{alpha inhibitors in patients with rheumatologic conditions. Archives of Dermatology, 143, 223-231. doi:10.1001/archderm.143.2.223

[12] Roux, C.H., Brocq, O., Leccia, N., et al. (2007) New-onset psoriatic palmoplantaris pustulosis following infliximab therapy: A class effect? The Journal of Rheumatology, 34, 434-437.

[13] Cohen, J.D., Bournerias, I., Buffard, V., et al. (2007) Psoriasis induced by tumor necrosis factor-alpha antagonist therapy: A case series. The Journal of Rheumatology, 34, 380-385.

[14] Pirard, D., Arco, D., Debrouckere, V. and Heenen, M. (2006) Anti-tumor necrosis factor alpha-induced psoriasiform eruptions: Three further cases and current overview. Dermatology, 213, 182-186. doi:10.1159/000095033

[15] Collamer, A.N., Guerrero, K.T., Henning, J.S. and Battafarano, D.F. (2008) Psoriatic skin lesions induced by tumor necrosis factor antagonist therapy: A literature review and potential mechanisms of action. Arthritis \& Rheumatism, 59, 996-1001. doi:10.1002/art.23835

[16] Palucka, A.K., Blanck, J.P., Bennett, L., et al. (2005) Cross-regulation of TNF and IFN-alpha in autoimmune diseases. Proceedings of the National Academy of Sciences of the United States of America, 102, 3372-3377. doi:10.1073/pnas.0408506102

[17] Aeberli, D., Seitz, M., Juni, P. and Villiger, P.M. (2005) Increase of peripheral CXCR3 positive $\mathrm{T}$ lymphocytes upon treatment of RA patients with TNF-alpha inhibitors. Rheumatology (Oxford), 44, 172-175. doi:10.1093/rheumatology/keh437

[18] Nestle, F.O., Conrad, C., Tun-Kyi, A., et al. (2005) Plasmacytoid predendritic cells initiate psoriasis through interferon-alpha production. The Journal of Experimental Medicine, 202, 135-143. doi:10.1084/jem.20050500

[19] Funk, J., Langeland, T., Schrumpf, E. and Hanssen, L.E. (1991) Psoriasis induced by interferon-alpha. British Journal of Dermatology, 125, 463-465. 


$$
\text { doi:10.1111/j.1365-2133.1991.tb14774.x }
$$

[20] van der Fits, L., van der Wel, L.I., Laman, J.D., et al. (2004) In psoriasis lesional skin the type I interferon signaling pathway is activated, whereas interferon-alpha sensitivity is unaltered. Journal of Investigative Dermatology, 122, 51-60.

\section{doi:10.1046/j.0022-202X.2003.22113.X}

[21] Gilliet, M., Conrad, C. and Geiges, M., et al. (2004) Psoriasis triggered by toll-like receptor 7 agonist imiquimod in the presence of dermal plasmacytoid dendritic cell precursors. Archives of Dermatology, 140, 1490-1495. doi:10.1001/archderm.140.12.1490 